\title{
SEBASTIÁN MUÑOZ, RUIZ DE LA IGLESIA Y FRANCISCO RIZI: UN NUEVO EJEMPLO DE LA CIRCULACIÓN DE MODELOS EN LA PINTURA DE LA SEGUNDA MITAD DEL SIGLO XVII EN MADRID
}

\author{
EdUARDo Lamas-Delgado \\ Institut Royal du Patrimoine Artistique (KIK-IRPA) Bruselas
}

\begin{abstract}
Este artículo presenta y estudia la estrecha relación, hasta ahora desconocida, entre el cuadro de altar del pintor madrileño Francisco Ignacio Ruiz de la Iglesia (1649-1703) para la villa de Casarrubios del Monte, concebido anteriormente por Sebastián Muñoz (h.1650-1690), y un dibujo del mismo asunto obra del pintor Francisco Rizi (1614-1685). Palabras clave: Pintura; Dibujo; Madrid; Barroco; Prácticas de taller; Circulación de modelos; Francisco Rizi; Sebastián Muñoz; Francisco Ignacio Ruiz de la Iglesia.
\end{abstract}

\section{SEBASTIÁN MUÑOZ, RUIZ DE LA IGLESIA AND FRANCISCO RIZI: ANOTHER EXAMPLE OF THE CIRCULATION OF PAINTING MODELS IN MADRID DURING THE SECOND HALF OF THE $17^{\mathrm{TH}}$ CENTURY}

This article reveals and elaborates upon the close relationship between the painted altarpiece by the Madrid artist Francisco Ignacio Ruiz de la Iglesia (1649-1703) for the village of Casarrubios del Monte, previously conceived by Sebastián Muñoz (c.1650-1690), and a drawing of the same subject by the painter Francisco Rizi (1614-1685).

Key words: Painting; drawing; Madrid; Baroque; Workshop practices; Circulation of models; Francisco Rizi; Sebastián Muñoz; Francisco Ignacio Ruiz de la Iglesia.

El cuadro de altar de la antigua iglesia parroquial de Casarrubios del Monte (fig. 1), el Martirio de san Andrés, una de las obras más importantes del pintor Francisco Ignacio Ruiz de la Iglesia (1649-1703), ha sido recientemente estudiado en un interesante artículo ${ }^{1}$. Como han señalado sus autores, su composición presenta una influencia evidente del cuadro de Rubens del mismo asunto (fig. 2) que decoraba el altar mayor de la capilla del Hospital de los Flamencos en Madrid². No obstante, quisiéramos atraer aquí la atención sobre un eslabón que ha pasado desapercibido en la cadena formal que va desde el cuadro del flamenco hasta el de Casarrubios.

Como ya se sabe, el cuadro de Casarrubios, firmado en 1696, había sido encargado en un principio a un amigo próximo del artista, el pintor Sebastián Muñoz (ca.1650-1690) que lo dejó inacabado al morir en un accidente ${ }^{3}$. Palomino nos dice que Muñoz había dejado "ya hecha la traza, y el cuadro imprimado, el cual ejecutó Don Francisco Ignacio por el mismo borroncillo"4. Aunque no se haya identificado el borroncillo o boceto de Muñoz, debemos tener por cierta la afirmación de Palomino y considerar que Ruiz de la Iglesia siguió su composición. Además de la presencia del borroncillo, hay que tener en cuenta, como ya señaló Angulo, que de las palabras del biógrafo se entiende que Muñoz también había dejado la composición ya dibujada en el lienzo imprimado5.

Los estudios preparatorios para el cuadro de Ruiz de la Iglesia, felizmente localizados por Zapata y Gómez Aragüete en la Casa de la Moneda, no ponen en duda esta afirmación. Se trata tan sólo de estudios parciales destinados a la concepción de ciertas figuras y en ningún caso de la composición general. Por tanto, ante la insistencia de Palomino en este detalle, se debe considerar a Muñoz como el autor de la composición que siguió Ruiz de la Iglesia.

Sin embargo, existe un nuevo elemento en la concepción de la obra que ha pasado inadvertido hasta ahora y que nos informa de otra importante fuente para la composición de Muñoz en el borron-

\footnotetext{
1 Zapata Fernández de la Hoz; Gómez Aragüete, 2012, pp. 17-36.

2 Vlieghe, 1972-1973, p. 89.

3 Zapata Fernández de la Hoz; Gómez Aragüete, 2012, p. 19.

${ }^{4}$ Palomino, 1947, pp. 1052 y 1115.

5 Angulo, 1979, pp. 385-387.
} 


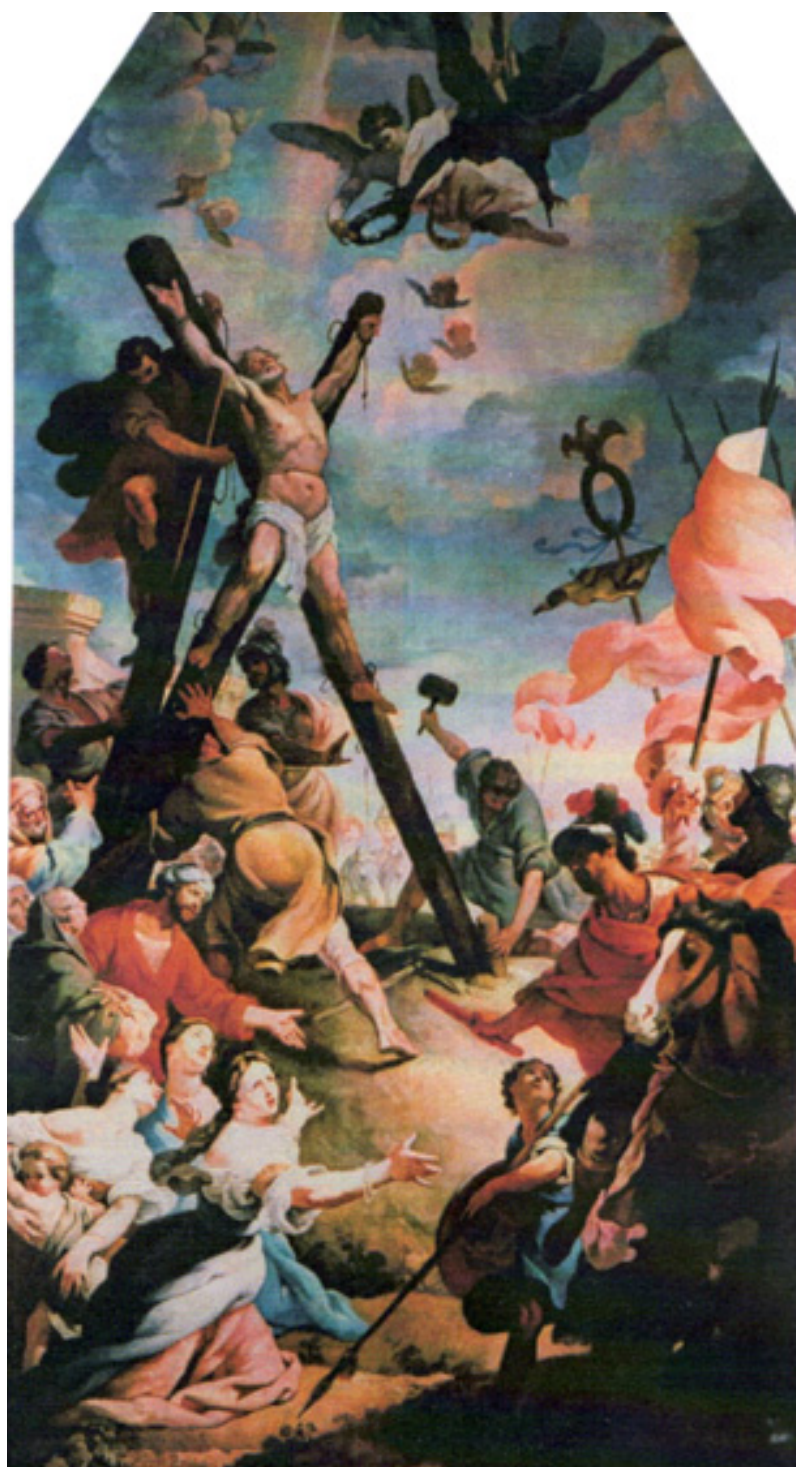

Fig. 1. Francisco Ignacio Ruiz de la Iglesia, Martirio de san Andrés, óleo sobre lienzo, 810 x $413 \mathrm{~cm}$. Casarrubios del Monte, iglesia parroquial.

cillo empleado por de la Iglesia. Como se decía, ésta deriva de la del lienzo de Rubens en el Hospital de los Flamencos de Madrid, entonces uno de los cuadros más célebres de Madrid ${ }^{6}$. Sin embargo, existe otro claro antecedente del cuadro de $\mathrm{Ca}$ sarrubios que muestra la deuda de Muñoz con un segundo artista. En efecto, el cuadro se inspira ampliamente, aunque invirtiéndola, de la composición del dibujo del mismo asunto que Jonathan Brown atribuyó a Francisco Rizi (1614-1685) en 1983 , y que por lo acabado de su presentación él ya consideró como un modello para un cuadro de altar $^{7}$ (fig. 3).

Pocos son los cambios introducidos en el cuadro de Muñoz-Ruiz de la Iglesia con respecto al modelo del dibujo, que tienden sobre todo a aligerar la composición. Como han señalado Zapata y Gómez Aragüete, la escena representada es aquélla en que los verdugos terminan de atar el Apóstol a la cruz ${ }^{8}$. Según la tradición, veintiún hombres le ataron, de los que Rizi hace figurar diecisiete $^{9}$. Les acompañan el procónsul romano Egeas a caballo y su esposa Maximila, que aparece implorando el perdón de Andrés en el primer término a la derecha en el dibujo. El cuadro de Casarrubios elimina dos de los personajes propuestos por Rizi: a saber, el soldado romano que aparece en primer término atrayendo con su brazo extendido la atención de Egeas sobre la petición de su esposa para que libere al supliciado, y los verdugos representados al pie de la cruz a la izquierda, remplazados por otra figura en el acto de levantar un mazo para clavarla al suelo. Otro elemento del dibujo que desaparece en el cuadro es el árbol situado detrás de la figura de Egeas a caballo, remplazado para dar un mayor desarrollo a la insignia romana y a la banderola. Por otra parte, el cuadro de Casarrubios introduce también una serie de cambios de posturas en las figuras

\footnotetext{
${ }^{6}$ Vosters, 1990, p. 182.

${ }^{7}$ Brown, 1983, p. 405 , no 12 , fig. 32.

${ }^{8}$ Zapata Fernández de la Hoz; Gómez Aragüete, 2012, p. 19.

9 Voragine, 1998, p. 14-15.
} 


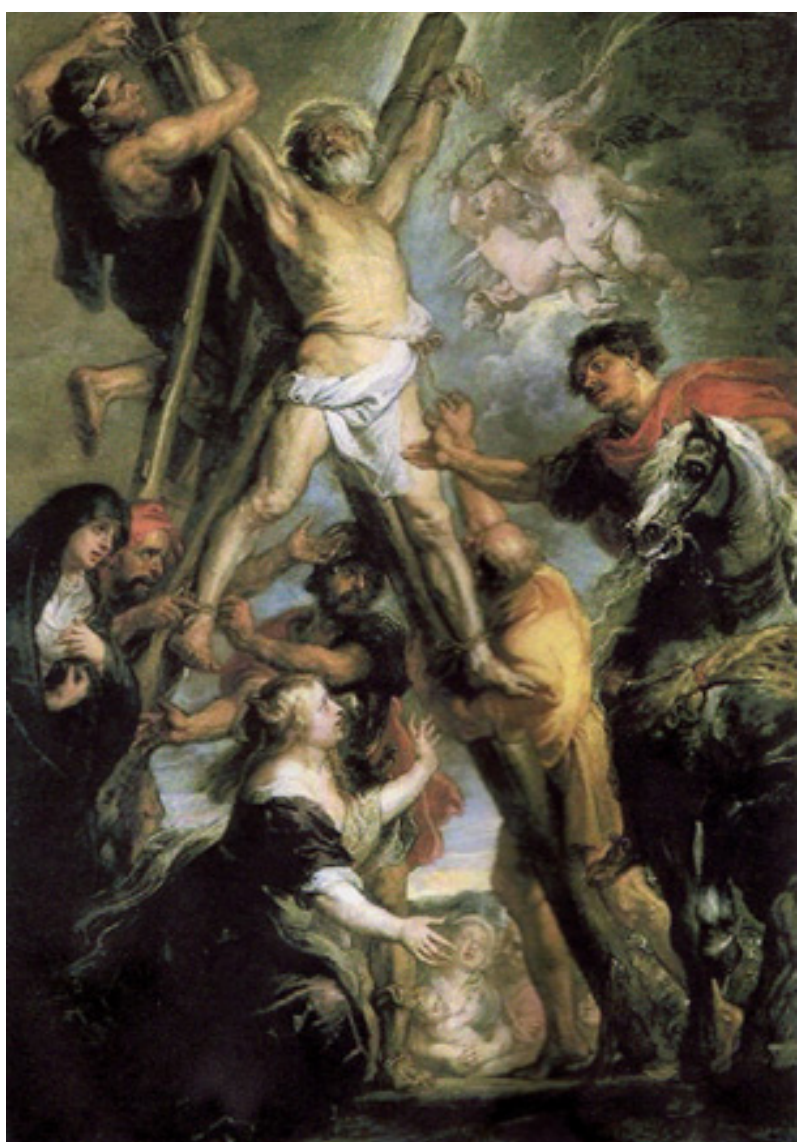

Fig. 2. Peter Paul Rubens, Martirio de san Andrés, óleo sobre lienzo, 306 x $216 \mathrm{~cm}$. Madrid, Fundación Carlos de Amberes. del primer término, así como en la parte superior del lienzo, donde dos ángeles mancebos presentan a san Andrés la corona y la palma del martirio, mientras que en el dibujo los lleva un grupo de cuatro angelotes.

Ante la evidente relación entre el dibujo de Rizi y el cuadro de Ruiz de la Iglesia conviene plantearse la relación de aquél con la Parroquia de San Andrés de Casarrubios del Monte. De hecho, sabemos que al menos veinticinco años antes del encargo del cuadro de altar a Muñoz, la iglesia ya había encargado la renovación de su altar mayor al ensamblador madrileño Pedro de la Torre (1596-1677). En 1676, un año antes de morir, el arquitecto-ensamblador afirmaba en su testamento que tenía pendiente de hacer el retablo mayor de la iglesia parroquial de Casarrubios, que se le habría encargado antes, y para el que había formado compañía con su sobrino Francisco de la Torre, y que probablemente nunca llegaron a realizar ${ }^{10}$.

La presencia de Pedro de la Torre en el proyecto de Casarrubios autoriza a albergar la hipótesis de la participación de Rizi, que fue uno de sus más estrechos colaboradores $^{11}$. En efecto, el pintor estuvo asociado a buena parte de sus proyectos, así como a algunos llevados a cabo por su socio y primo José de la Torre $^{12}$. De esta asociación se conocen diversos ejemplos: la entrada de la reina Mariana en Madrid en $1649^{13}$, el retablo de la iglesia de Santiago en Madrid (1648-1658) $)^{14}$, el retablo de la Expectación en el convento de los Trinitarios Calzados de Madrid (1652-1656) ${ }^{15}$, o el la parroquial de Vallecas $(1669-1670)^{16}$, entre otros. En sus proyectos juntos, era muy habitual que Rizi participase en la ejecución de las trazas que el ensamblador presentaba a los comitentes, especialmente cuando se trataba de incluir modelos para pinturas. ¿Pudo entonces el dibujo del Martirio de san Andrés ser concebido por Rizi para figurar

${ }^{10}$ El testamento habla de retablo con su custodia, de que se deduce que estaba destinado al altar mayor de la iglesia. Véase: TOVAR MARTín, 1975, pp. 185-186.

${ }^{11}$ El autor ultima un estudio donde se analiza la colaboración entre ambos artistas.

12 Agulló, 1997.

13 Brown; Banner; Galassi, 2010, pp. 103-106.

${ }^{14}$ Cruz Yábar, 2005, pp. 155-178.

15 Lamas-Delgado, 2013.

${ }^{16}$ Díaz Fernández, 2004, pp. 149-172.

Arch. esp. arte, LXXXVI, 344, OCTUBRE-DICIEMBRE 2013, 345-362, ISSN: 0004-0428 


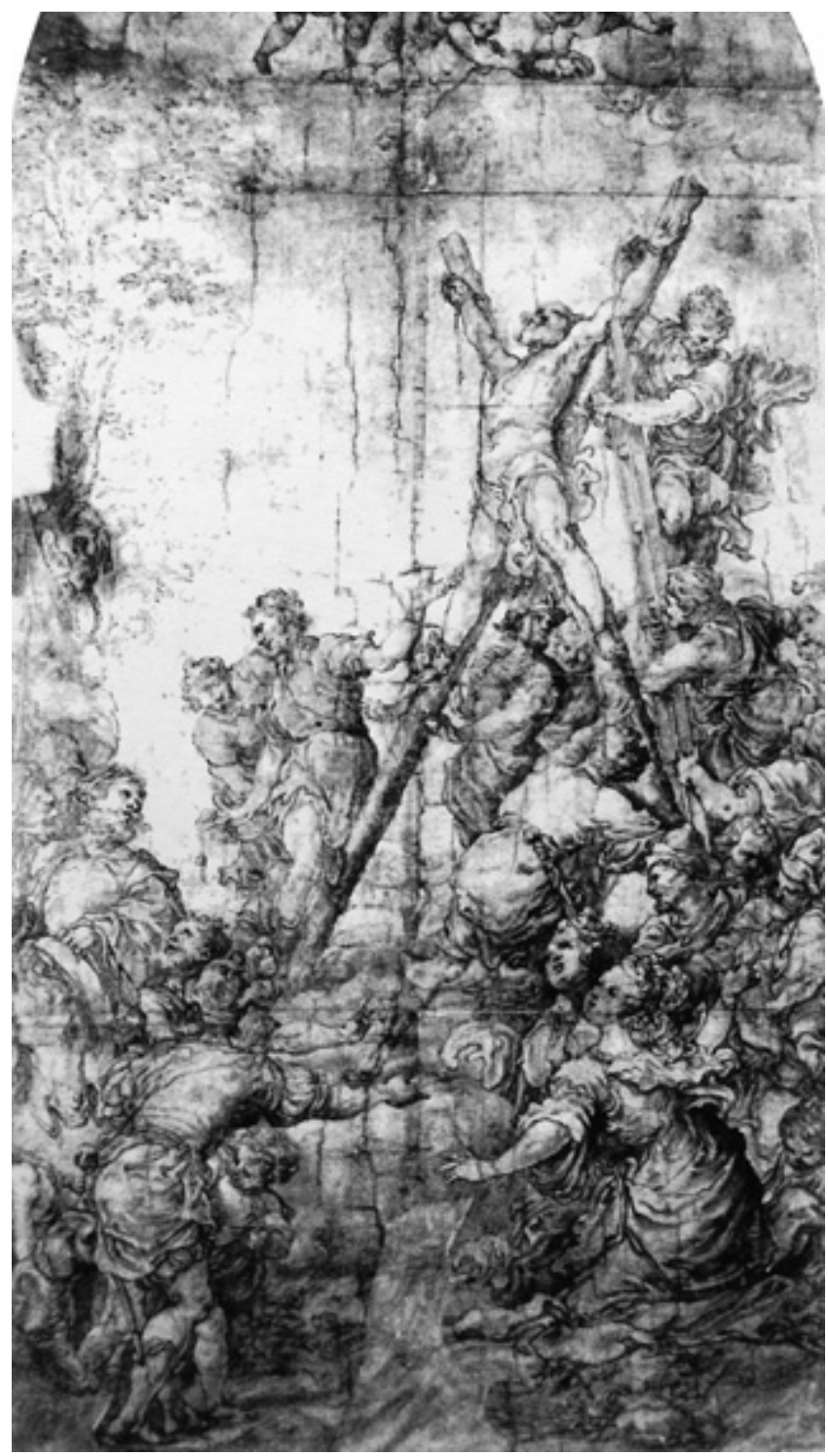

Fig. 3. Francisco Rizi, Martirio de san Andrés, pluma, tinta parda y aguada rojiza sobre papel con cuadriculado a lápiz, 598 x $298 \mathrm{~mm}$. Colección privada.

junto a la traza que los de la Torre presentaron para el contrato del retablo de Casarrubios? Es muy probable, así como que la Parroquia de Casarrubios lo hubiese conservado, lo que justificaría que Sebastián Muñoz lo emplease como modelo años más tarde para elaborar su composición para el cuadro de altar definitivo.

En tal caso, la Parroquia se habría dirigido a Rizi y de la Torre en un primer momento, antes de solicitar los servicios de Sebastián Muñoz más de veinticinco años más tarde. Si Rizi llevó a cabo su dibujo para atender una comisión para Casarrubios, cabe contemplar dos posibilidades: o bien el encargo, realizado en una fecha indeterminada, pero antes de 1676, nunca llegó a materializarse. O bien, 
éste le llegó poco antes de morir en 1685, quedando el proyecto inacabado, de modo que éste pasó unos pocos años más tarde a Sebastián Muñoz, con las circunstancias que ya conocemos. Proponemos rechazar el segundo caso, por parecernos el menos probable. En primer lugar, resultaría sorprendente que Palomino, que conoció a Rizi, no hablase de este encargo; por otro, el estilo del dibujo, según veremos no corresponde con la manera de hacer de Rizi en el último período de su vida.

En principio, el estilo del dibujo de Rizi nos permite proponer una fecha de ejecución no muy alejada de la década que media entre 1649 y 1659, debido a las similitudes que presenta con los dibujos preparatorios de la Biblioteca Nacional que el artista hizo para la entrada triunfal de la reina Mariana de Austria en Madrid en 1649, así como con su composición para el cuadro de altar de Fuente el Saz, de 1655, y con su dibujo preparatorio para el techo del Salón de los Espejos en el Alcázar, de hacia 1659, conservado en el Museo de Valencia ${ }^{17}$. Por un lado, el modo de concebir las figuras y los volúmenes obtenidos a través de las aguadas son los mismos que los de los dibujos para la entrada de $1649^{18}$. Por otro, el revoloteo de los drapeados de las figuras es el mismo que Rizi emplea a menudo en sus cuadros de esos mismos años: como en el grupo de las santas mujeres del Expolio de Cristo de la Almudena, de 1651, en el san Gabriel de la Anunciación de Plasencia de 1655, o en el mismo dibujo de Valencia.

El dibujo de Rizi ya se puso en relación con el cuadro de la Crucifixión de san Pedro en Fuente el Saz cuando fue expuesto en París en $1991^{19}$. El modo de distribuir las masas en la superficie de la hoja es muy similar al de la pintura, cuya composición se articula también en torno a la línea diagonal formada por la cruz. En el dibujo, a la izquierda, la figura truncada del caballo en escorzo del procónsul romano Egeas cierra la composición de una manera similar a la del prefecto romano montado a caballo en la derecha del cuadro de Fuente el Saz.

Aunque invertida, como decíamos, la composición del dibujo se relaciona también estrechamente con el cuadro de Rubens. Expuesto en su capilla desde 1640, Rizi ya había tenido la ocasión de estudiarlo con atención para su San Andrés de 1646 en el Museo del Prado ${ }^{20}$. Como en aquella ocasión, Rizi se sirve del modelo para crear una nueva composición, cuyas referencias al maestro de Amberes debieron de ser muy apreciadas por sus contemporáneos aficionados a la pintura.

La adaptación que hizo Muñoz de la composición de Rizi, aparte de la inversión que le devuelve la orientación que le había dado Rubens, confiere una mayor legibilidad al conjunto, al simplificar el número de figuras. Curiosamente, están documentadas otras dos intervenciones similares en otras dos composiciones creadas por Rizi, quien se habría caracterizado por un gusto por la acumulación de masas en la distribución de los personajes. Así parece que hizo Juan Carreño (1614-1685) en el cuadro de altar para Pamplona, que se basa en un dibujo de Rizi $^{21}$, y Ximénez Donoso (ca 1632-1690) en el de la iglesia de San Ginés de Madrid, cuando, según Palomino, "habiendo muerto Rizi, y pareciendo que estaba algo confuso dicho cuadro, lo retocó" 22 .

Si realmente el dibujo de Rizi se hizo con destino al retablo de Casarrubios, es muy probable que la Parroquia lo hubiese guardado con el contrato a Pedro de la Torre. Años más tarde, en cualquier caso más de veinticinco, al retomar la obra del retablo, la Parroquia lo podría haber puesto a disposición de Muñoz como orientación de lo que ésta deseaba.

\footnotetext{
${ }^{17}$ Francisco Rizi, Júpiter entrega la caja a Pandora, pluma y aguada parda sobre papel verjurado, 336 x $285 \mathrm{~mm}$. Valencia, Museo de Bellas Artes de San Pío V.

18 PÉrez SÁnChez, p. 245.

${ }^{19}$ STEIN, 1991, nº 59 .

${ }^{20}$ Lamas-Delgado, 2011, p. 7.

${ }^{21}$ Gérard Powell; Ressort, pp. 153-157

${ }^{22}$ Palomino, p. 1017.
} 
No obstante, quisiéramos señalar otro posible origen para el dibujo de Rizi, si bien lo creemos menos probable. Así, podría estar en relación con la iglesia parroquial de San Andrés en Madrid, cuya capilla mayor se estaba ultimando durante los años que hemos propuesto aquí para fechar el dibujo. Su retablo, donde figuró una escultura de bulto redondo del Apóstol, fue finalmente realizado por el arquitecto Juan de Lobera $(\uparrow 1681)$, con quien se firmó un contrato para llevarlo a cabo en octubre de $1659^{23}$. Sin embargo, sabemos que Alonso Cano (1601-1667) realizó hacia 1657 una traza alternativa, conservada en el Museo del Prado, y donde se preveía un gran cuadro con el martirio del santo ${ }^{24}$. Como Pedro de la Torre estuvo asimismo trabajando en proyectos para la reforma de la iglesia, cabe albergar la posibilidad de que él presentase una tercera traza para el retablo en colaboración con Rizi, donde también se habría propuesto un gran cuadro de altar con el martirio del santo. De hecho, la década de 1650 es en la que ambos artistas colaboran más intensamente. Recuérdese que en esas mismas fechas preparaban juntos el retablo de Santiago, y que Rizi realizaba el de Fuente el Saz con Jusepe de la Torre, el pariente y socio habitual de Pedro ${ }^{25}$.

En tal caso, ¿cómo es posible que el dibujo de Rizi llegase a manos de Sebastián Muñoz tantos años después? Palomino nos dice que en el taller de Rizi, donde el dibujo era práctica corriente, su discípulo Claudio Coello (1642-1693) "tenía gran cuidado de recoger [los dibujos de su maestro] y juntarlos y estudiar en ellos" sus enseñanzas ${ }^{26}$. El dibujo del Martirio de san Andrés podría haber sido objeto de colección por parte de Coello quien, como cualquier artista de la época, siempre estaría interesado en disponer de modelos con los que llevar a cabo sus propias composiciones. Así, el dibujo también podría haber llegado a manos Sebastián Muñoz a través del propio Coello, que fue su maestro.

En cualquier caso, sea cual sea el camino seguido por el dibujo de Rizi para llegar a manos de Muñoz, no nos cabe duda de que éste le sirvió para elaborar la composición del cuadro de Casarrubios. Más tarde, Ruiz de la Iglesia supo adaptarla a su propio estilo con gran talento, hasta el punto de llegar a crear una de sus mejores obras.

Aquí se repetiría el caso del cuadro de Giordano (1634-1705) de la Aparición de la Virgen a san Fernando con santa Úrsula y las once mil vírgenes del Escorial, de hacia 1695-96, que se basaba en un bosquejo inacabado de Carreño, de al menos diez años antes, pero reinterpretado a través de un dibujo de Coello ${ }^{27}$.

Este nuevo ejemplo de circulación de modelos entre los maestros del barroco madrileño debe servir para atraer nuestra atención sobre la problemática del proceso creativo de las obras de arte y de las prácticas de taller durante este período, cuya realidad presenta una complejidad mayor de la que la historiografía venía presentando tradicionalmente. No obstante, la ruptura con la visión romántica del artista y un nuevo interés por la colaboración entre maestros, talleres y disciplinas ya se va abriendo paso en la historiografía en los últimos años ${ }^{28}$. A medida que aumente nuestro conocimiento sobre la obra realizada por los maestros madrileños, irán saliendo a la luz nuevos juegos de influencias y de interrelaciones hoy desapercibidas, lo que seguramente nos permitirá abarcar con más tino la coherencia y la personalidad definida que caracterizan el movimiento barroco en Madrid.

\footnotetext{
23 Tovar Martín, 1975, pp. 272-273.

${ }^{24}$ Rodríguez Rebollo, 2007, pp. 452-458.

${ }^{25}$ Barrio MoYa.

${ }^{26}$ Palomino, 1947, p. 1059.

${ }^{27}$ Lamas Delgado, 2006.

${ }^{28}$ En el contexto español, véanse interesantes reflexiones sobre la cuestión en: GuTIÉRREz PASTOR, 1992 y 2006, y en Navarrete Prieto.
}

Arch. esp. arte, LXXXVI, 344, octUBRE-DICIEMBRE 2013, 345-362, ISSN: 0004-0428 


\section{BIBLIOGRAFÍA}

Agulló Cobo, Mercedes, "Pedro, José, Francisco y Jusepe de la Torre, arquitectos de retablos", Anales del Instituto de Estudios Madrileños, 37, 1997, pp. 25-70.

Agulló Cobo, Mercedes, “Addenda a Pedro de la Torre”, Anales del Instituto de Estudios Madrileños, 37, 1998, pp. 117-194.

Angulo, Diego, "Francisco Ignacio Ruiz de la Iglesia”, Archivo Español de Arte, 52, 208, 1979, pp. 467-504.

Barrio Moya, José Luis, "José de la Torre y Francisco Ricci, autores del retablo mayor de la iglesia de Fuente del Saz del Jarama”, Anales Complutenses, XII, 2000, pp. 43-54.

Brown, Jonathan, "Selected drawings by Spanish Baroque Masters", Master Drawings, 21-4, 1983, pp. 401-407.

Brown, Jonathan; Banner, Lisa A.; Galassi, Susan Grace, ed., The Spanish Manner: Drawings from Ribera to Goya, New York, The Frick Collection/Scala Publishers, 2010.

Cruz Yábar, José María, "Los retablos de la parroquia de Santiago de Madrid, Pedro de la Torre, Sebastián de Benavente y Alonso Cano", Anales del Instituto de Estudios Madrileños, 45, 2005, pp. 155-178.

Díaz Fernández, Antonio José, "El retablo mayor de la parroquial de Vallecas (Madrid): una arquitectura desaparecida de Pedro y Francisco de la Torre (1672)", Espacio, Tiempo y Forma, Serie VII, Historia del arte, 17, 2004, pp. 149-172.

Gérard Powell, Véronique; Claudie Ressort, Musée du Louvre. Département des peintures. Catalogue. Écoles espagnole et portugaise, Paris, RMN, 2002.

Gutiérrez Pastor, Ismael, "Francisco Rizi y Claudio Coello: a propósito de la anécdota de Palomino sobre el Retablo de la Parroquia de Santa Cruz de Madrid", Anuario del Departamento de Historia y Teoría del Arte (UAM), 4, 1992, pp. 231-238.

Gutiérrez Pastor, Ismael, "Géneros y colaboraciones en la pintura madrileña de la segunda mitad del siglo XVII: a propósito de los lienzos de Bartolomé Pérez en el retablo mayor de Santa María de Gumiel del Mercado (Burgos)", Anuario del Departamento de Historia y Teoría del Arte (UAM), 18, 2006, pp. 107-124.

Lamas Delgado, Eduardo, "Un dibujo de Claudio Coello para un cuadro de Luca Giordano", Anuario del Departamento de Historia y Teoría del Arte (UAM), 18, 2006, pp. 97-106.

Lamas Delgado, Eduardo, “'Un autre Rubens': l'influence du rubénisme dans la peinture courtisane espagnole à travers l'exemple de l'œuvre de Francisco Rizi (1614-1685)", Revue Belge d'Histoire de l'Art et Archéologie=Belgisch Tijdschrift voor Oudheidkunde en Kunstgeschiedenis, 80-1, 2011, pp. 179-196.

Lamas Delgado, Eduardo, "Partnership between painters and sculptors in 17th-century Spain: on model drawings by Francisco Rizi for an altarpiece of the Expectant Virgin”, RIHA Journal, 2013.

Navarrete Prieto, Benito, "Flandes e Italia en la pintura barroca madrileña: 1660-1700”, en: Navarrete Prieto, Benito; Zapata Fernández de la Hoz, María Teresa; Martínez Ripoll, Antonio, Fuentes y modelos de la pintura barroca madrileña, Madrid, Arco Libros, 2008, p. 11-103.

Palomino, Antonio, Museo pictórico y escala óptica, Madrid, Aguilar, 1947.

Pérez Sánchez, Alfonso E., Historia del dibujo en España. De la Edad Media a Goya, Madrid, 1986.

Rodríguez Rebollo, Ángel, “A propósito de Alonso Cano: el dibujo para el retablo de San Diego de Alcalá y su homónimo para San Andrés”, In Sapientia Libertas: escritos en homenaje al Profesor Alfonso E. Pérez Sánchez, Madrid, Museo Nacional del Prado, 2007.

Stein, Adolphe, Dessins espagnols, cat. exp., Paris, Galerie Gismondi, 1991.

Tovar Martín, Virginia, Arquitectos madrileños de la segunda mitad del siglo XVII, Madrid, Instituto de Estudios Madrileños, 1975.

Vlieghe, Hans, Corpus Rubenianum Ludwig Burchard: Part VIII: Saints, Brussels-London, Arcade/Phaidon, 1972-1973.

Voragine, Jacques de la, La Légende dorée, Paris, Seuil, 1998.

Vosters, Simon A., Rubens y España: estudio artístico literario sobre la estética del barroco, Madrid, Cátedra, 1990.

Zapata Fernández de la Hoz, María Teresa; Gómez Aragüete, Juan Carlos, "Nuevas aportaciones a la obra de Francisco Ignacio Ruiz de la Iglesia (1649-1703)”, Archivo Español de Arte, 85, 337, 2012, pp. 17-36.

Fecha de recepción: 23-III-2012

Fecha de aceptación: 28-XI-2012

Arch. esp. arte, LXXXVI, 344, OCTUBRE-DICIEMBRE 2013, 345-362, ISSN: 0004-0428 